\title{
SIMPLIFIED MORTON EFFECT ANALYSIS FOR SYNCHRONOUS SPIRAL INSTABILITY
}

\author{
Brian T. Murphy \\ Center for Electromechanics \\ University of Texas \\ Austin, Texas, USA
}

\author{
Joshua A. Lorenz \\ Kato Engineering \\ Mankato, Minnesota, USA
}

\section{ABSTRACT}

A simplified analytical approach for modeling the synchronous instability phenomenon known as the Morton effect is presented. The analysis is straight forward and easily applied to any rotor supported on fluid film bearings. The analysis clarifies the interaction of three distinct machine characteristics which combine to create a case of the Morton effect. Some example calculations are shown illustrating the possible types of spiral vibration.

In addition, an analytical approach is described for estimating the magnitude of the shaft temperature difference in a journal bearing as a direct function of the shaft orbit. It is significant that this method can readily be applied to any type of journal bearing, from plain sleeve bearings to tilting pad bearings. Example calculations using the method are shown.

\section{INTRODUCTION}

It has been known for many years that thermal temperature gradients across a rotor can alter the amplitude and phase of synchronous vibration. This is due to bowing of the shaft caused by a temperature difference between one side of the shaft and the other. The earliest published recognition of this phenomenon was by Newkirk [1], where the temperature difference was a result of frictional heating from rubbing of the rotor on non-rotating elements of the machine. A rub of this type could involve labyrinth seals, armatures, impellers, slip rings, etc. Residual rotor imbalance produces vibration, which produces a rub, which generates heat locally on the shaft surface, which bows the shaft, which causes additional imbalance, which leads to increased vibration and heat, and so on. This could be described as a type of thermal runaway.

Another very different way that a temperature difference in the shaft can be produced is via non-uniform viscous shearing action in the oil film of a journal bearing. This particular type of thermal action has come to be referred to as the Morton Effect [2]. An excellent and thorough review of literature on this topic is given by De Jongh [3]. 
The Morton effect is a thermal condition which exists to various extents in all fluid film journal bearings. Figure 1 depicts a cylindrical sleeve bearing with an orbiting journal. An essential underlying condition of the Morton effect is that the shaft orbital motion is synchronous with rotation. In figure 1a the shaft is at its closest approach to the stationary bearing liner. In this position the maximum amount of viscous shearing heat is generated at the location of minimum film thickness. This is a fundamental aspect of hydrodynamic lubrication. This particular spot on the shaft surface is marked with a red circle and subsequently referred to as the "high spot". The four successive orbital positions displayed in figure 1 (every 90 degrees of shaft rotation) show that the "high spot" is the only spot on the shaft that experiences the smallest possible film thickness (as well as the smallest time average film thickness) and its accompanying maximum heat input, during the course of one complete shaft orbit and revolution. The result is that different regions of the shaft surface are exposed to different amounts of time averaged heat input during a single orbit and revolution. This produces a fixed (relative to the shaft) temperature difference across the shaft. The temperature difference is proportional to the size of the orbit. When the orbit size is zero, and the shaft only spins, all regions of the shaft surface are exposed to identical amounts of time averaged heat input during one orbit and revolution. In this case there is no temperature difference created. As orbit size increases, the variation in local time averaged heat input becomes greater. A temperature difference across the shaft which increases as orbit size increases, results from this variation. The temperature differential can be thought of as being fixed with respect to the moving shaft coordinates and the temperature differential is able to penetrate deep into the material of the shaft. This type of thermal gradient is not to be confused with the time varying 'skin effect' temperature differentials which are present on the surface of the shaft and are a result of the shaft passing though a lubricant field with spatially varying temperatures once per revolution [4].

Most rotating machines are not affected by the aforementioned temperature difference in a journal bearing. Some particular machine configurations, however, are sensitive. A quite common trait is a large amount of overhung weight outboard of one or both bearings. Examples include turbochargers and turboexpanders with large overhung impellers, high-power compressors with large heavy coupling hubs, and electrical generators with overhung exciters. Shaft bowing at the bearing throws the overhung weight out of balance. If the machine is running at a speed where vibration amplitude at the bearing is sensitive to imbalance of the overhung weight, then conditions that produce the Morton effect may be satisfied.

\section{NOMENCLATURE}
A Balance sensitivity vector (micron/gm-mm)
B Temperature coefficient vector $\left({ }^{\circ} \mathrm{C} /\right.$ micron $)$ 
C Bow coefficient vector (gm-mm. $\left./{ }^{\circ} \mathrm{C}\right)$

D Effective thermal damping

E Effective thermal stiffness

L Bearing axial length (meters)

$\mathrm{L}_{\mathrm{w}} \quad$ Axial distance from bearing to overhung mass (meters)

M Amount of overhung mass (kg)

$\mathrm{R} \quad$ Shaft radius at the bearing (meters)

s Complex eigenvalue (1/seconds)

$\mathrm{T} \quad$ Shaft temperature difference vector $\left({ }^{\circ} \mathrm{C}\right)$

V Vibration vector, or high spot (micron)

U Imbalance vector, or heavy spot (gm-mm)

$\alpha \quad$ Coefficient of thermal expansion

$\tau \quad$ Time constant (see Eqn. 6)

\section{ANALYSIS}

The Morton effect will be mathematically described using simple relationships. Each relationship is a linear one among vector quantities. An assumption inherent in this approach is that the thermal response of the rotor system be "infinitely slow" compared to the rotordynamic response of synchronous vibration. In other words, rotor vibration is assumed to always be quasi steady state.

In what follows all vector quantities have amplitude and phase angle. The phase angles are with respect to a once-per-revolution phase marker assumed to be at a fixed position on a rotating shaft. Such vector quantities are typically measured with modern day vibration analyzers. The phase angle sign convention can be either leading or lagging, but must be consistent throughout.

\section{Basic Vibration/Balance Relationship}

This is identical to the basic formula of influence coefficient balancing. It is a linear relationship between imbalance and vibration. In this analysis the calculated vibration is analogous to that which would be measured with shaft displacement sensors located in or near the bearings. 


$$
\vec{V}=\vec{A} \vec{U}
$$

where

$$
\begin{aligned}
& \vec{V}=\text { high spot, or vibration vector } \\
& \vec{U}=\text { heavy spot, or imbalance vector } \\
& \vec{A}=\text { influence coefficient vector }
\end{aligned}
$$

The vibration vector $\vec{V}$ serves to locate the "high spot" on the shaft surface. As previously mentioned, this is the spot on the shaft which comes closest to the bearing. The imbalance vector $\vec{U}$ serves to locate the "heavy spot" on the shaft. If the imbalance were in the form of a weight placed on the shaft, $\vec{U}$ would specify the magnitude and circumferential location of the weight. The vector quantity $\vec{A}$ is the sensitivity of vibration to imbalance.

\section{Steady State Thermal Relationship}

This locates the "hot spot" in relation to the position of the high spot, and also computes the magnitude of the temperature difference around the shaft. In the case of a sleeve bearing, this is determined by analyzing the bearing fluid film for a given synchronous whirl orbit and revolution to compute the heating and cooling around the shaft, and the dissipation of heat into and out of the shaft. The result of the calculations being the number of degrees of circumferential temperature difference (shaft delta T), plus the location of the hot spot on the shaft, relative to the high spot. In lieu of analysis, thermocouples embedded in the shaft could also be used to measure this directly. This is a steady state condition, and the resulting temperature difference is assumed to be linearly proportional to the size of the shaft orbit. Published experimental results from test rigs have shown that in journal bearings, hot spots lag high spots. In figure 1, the hot spot is being displayed as if it were coincident with the high spot.

$$
\begin{aligned}
& \vec{T}_{s s}=\vec{B} \vec{V} \\
& \vec{T}_{s s}=\text { steady state temperature vector } \\
& \vec{B}=\text { temperature coefficient vector }
\end{aligned}
$$

The vector quantity $\vec{B}$ is the sensitivity of shaft temperature difference to vibration. An analysis to rigorously compute values for shaft hot spot phase angle and magnitude of circumferential temperature difference is quite complex. This involves solving the problem of hydrodynamic fluid film lubrication with cavitation, thermal affects, and orbit motion, simultaneously with a transient heat 
transfer analysis of the fluid, bearing shell, and shaft. This has been done both analytically [2] and numerically [5] for specific bearings, but the applicability is limited to those specific bearings. It is apparent that a simplified method to make these calculations more easily for all bearing types is highly desirable.

\section{Basic Imbalance/Temperature Relationship}

This is determined by analyzing the shaft for how much it bows due to a temperature difference between hot and cold sides of the

shaft, and determining how much imbalance is thereby produced. This provides the value for $\vec{C}$ appearing in the following expression.

$$
\vec{U}=\vec{U}_{o}+\vec{C} \vec{T}
$$

where

$$
\begin{aligned}
& \vec{U}=\text { total imbalance vector } \\
& \vec{U}_{o}=\text { initial mechanical imbalance vector } \\
& \vec{T}=\text { hot spot, or temperature vector } \\
& \vec{C}=\text { bow coefficient vector }
\end{aligned}
$$

The vector quantity $\vec{C}$ is the sensitivity of imbalance to shaft temperature difference. In general, the angle for $\vec{C}$ will be either 0 or 180 degrees, depending upon which way the rotor gets thrown out of balance by the thermal bow. A rigorous analysis to compute $\vec{C}$ could be done as a steady state calculation with a structural FE model of the shaft. A prescribed temperature profile in the bearing would be applied, and the ensuing bow calculated. The amount by which the overhung weight is displaced from the shaft centerline is used to determine $\vec{C}$. As an example, consider a bearing having axial length $\mathrm{L}$ and diameter D. It can be shown (Dimarogonas [6]) that by assuming a temperature gradient which is linear and symmetric across the shaft, and uniform along the length of the bearing, the following amount of imbalance is produced at the center of gravity of the overhung weight.

$$
U_{t h}=M \frac{\alpha T}{R} L L_{w}
$$




\section{Transient Thermal Relationship}

This equation models how quickly the hot spot moves around the shaft. That is, the concept of the hot spot moving from where it is currently, to where it wants to be. The hot spot wants to be at its steady state position $\vec{T}_{s s}$, and will continuously try to move toward that spot. The rate of hot spot motion is determined by the thermal time constant of heat flow through the shaft, among other things. It will be seen below that the Morton effect stability (or lack thereof) is independent of this time constant.

$$
D \overrightarrow{\dot{T}}+E\left(\vec{T}-\vec{T}_{s s}\right)=0
$$

or

$$
\tau \overrightarrow{\dot{T}}+\vec{T}=\vec{T}_{s s}
$$

where

$$
\begin{aligned}
& E=\text { thermal "stiffness" } \\
& D=\text { thermal "damping" } \\
& \dot{\vec{T}}=\text { thermal velocity or time derivative of } \mathrm{T} \\
& \tau=\frac{D}{E}=\text { thermal time constant }
\end{aligned}
$$

The "thermal velocity" corresponds to the hot spot moving around the shaft. A thermal velocity of zero implies the hot spot is at a fixed location on the shaft. This is another situation where a rigorous analysis to compute the transient movement of the hot spot around the orbiting shaft is quite involved. Similar to the complexities involved in the calculations required for hot spot phase angle and temperature difference, calculations for the transient movement of the hot spot around the orbiting shaft also involve the additional coupling of rotordynamic and structural analyses (see for example, [7]). It will be shown below that the Morton instability can be predicted without requiring knowledge of the thermal velocity or thermal time constant.

The preceding expressions are combined as follows:

$$
\begin{aligned}
& \tau \overrightarrow{\dot{T}}+\vec{T}-\vec{B} \vec{V}=0 \\
& \tau \overrightarrow{\dot{T}}+\vec{T}-\vec{B}(\vec{A} \vec{U})=0 \\
& \tau \overrightarrow{\dot{T}}+\vec{T}-\vec{B} \vec{A}\left(\vec{U}_{o}+\vec{C} \vec{T}\right)=0
\end{aligned}
$$




$$
\begin{aligned}
& \tau \overrightarrow{\dot{T}}+\vec{T}-\vec{B} \vec{A} \vec{C} \vec{T}=\vec{B} \vec{A} \vec{U}_{o} \\
& \tau \overrightarrow{\dot{T}}+(1-\vec{B} \vec{A} \vec{C}) \vec{T}=\vec{B} \vec{A} \vec{U}_{o}
\end{aligned}
$$

Starting from an assumed initial condition for $\vec{T}$, Equation (7) allows calculation of the shaft temperature difference, and its location on the shaft, as a function of time. The expression applies to any constant value of rotor speed. The transient result for vector $\vec{T}$ can then be used to evaluate vector results for $\vec{U}$ and $\vec{V}$ by using Equations (1) and (3).

Equation (7) is seen to be a single first order differential equation with constant coefficients, and so its solution is a complex exponential with a single eigenvalue, $s$, expressed as:

$$
\begin{aligned}
& \vec{T}(t)=\vec{T} e^{s t} \\
& s=\frac{\vec{B} \vec{A} \vec{C}-1}{\tau}
\end{aligned}
$$

From physical considerations, the time constant $\tau$ is always a positive real value. The sole requirement for stability is that the real part of $s$ not be positive, which leads to:

$$
\operatorname{Re}(\vec{B} \vec{A} \vec{C}) \leq 1 \text { for stability }
$$

This shows that stability does not depend on the time constant, but only the three sensitivity vectors. Larger values of each of the three sensitivities, $\vec{A}, \vec{B}$ and $\vec{C}$ ought to make a the Morton effect instability more likely. However, the angles associated with these sensitivity vectors also influence stability. Even if the sensitivities are all large in magnitude, the rotor will still be stable if the three angles place the product of $\vec{B} \vec{A} \vec{C}$ to the left of +1 on the real axis of the complex plane. As rotor speed changes, the angle of $\vec{A}$ is the one that would tend to vary the most. The angle of $\vec{C}$ typically would not change at all. The angle of $\vec{B}$, for journal bearings, likely stays in a range of approximately 0 to 60 degrees (lagging).

As speed approaches a critical speed, both the magnitude and angle of $\vec{A}$ will be changing rapidly. The stability condition Equation (10) tells us that if the real part of the vector product of the three sensitivities is less than 1, the system is always stable. When stable, the transient thermal solution to the vibration prediction equations is a converging spiral (as viewed on a polar plot of vibration) which settles to a steady operating point. The steady vibration operating point is a blend of mechanical and thermal (but constant) imbalance. When unstable, the vibration response spiral diverges (i.e. thermal runaway). When on the boundary between 
stability and instability, the vibration response is a circle centered on the point of the corresponding stable solution. The size of the circle is a function of the initial condition. Figure 2 illustrates these three cases.

The rate at which the hot spot and high spot travel around the bearing journal is established by the imaginary part of the eigenvalue having units of radians/second. The rate is seen to be dependent not only on the thermal time constant, but also each of the sensitivity vectors.

$$
\frac{\operatorname{Im}(\vec{B} \vec{A} \vec{C})}{\tau}
$$

When the Morton effect is significant but stable, some interesting observations can be made concerning the steady state vibration behavior of a machine. First, in the absence of any thermal bow, the steady state vibration vector would be:

$$
\vec{V}_{s s}=\vec{A} \vec{U}_{o}
$$

With a stable thermal bow, this becomes (by setting $\dot{\vec{T}}=0$ in Equation (7)):

$$
\vec{V}_{s s}=\frac{\vec{A}}{1-\vec{A} \vec{B} \vec{C}} \vec{U}_{o}
$$

The thermal sensitivity of the machine is seen to modify the steady state vibration. Thus, even when stable, the steady state vibration vector could be excessive, and damage or trip the machine. Just being stable does not guarantee acceptably smooth vibration performance. Also, observe that in a machine which is especially sensitive to the Morton effect, the product $\vec{B} \vec{A} \vec{C}$ may have a magnitude much greater than one. In this situation, equation (13) indicates that the Morton effect will actually attenuate synchronous vibration. As speed increases, and an unstable machine approaches the Morton instability threshold, synchronous vibration could become very small before becoming unstable. Such behavior has been observed in a machine where 3 nominally identical units were built and tested, and all exhibited the Morton instability. The unit with by far the smallest synchronous vibration exhibited the most rapidly divergent unstable behavior. This will be a topic of a forthcoming paper.

The forgoing discussion suggests that a the Morton effect instability can be predicted with readily available rotordynamic analytical tools if only there were practical means of estimating the temperature sensitivity vector $\vec{B}$. Estimation of the temperature coefficient vector could be done if a way to estimate shaft temperature difference as a function of journal orbit were readily available 
for any type of bearing. Another practical approach to this problem is to perform tests to measure shaft temperature difference for a range of bearing designs (see for example [3] and [8]).

\section{CALCULATION METHOD FOR SHAFT DELTA T}

This section describes an approximate analytical approach for estimating the magnitude of shaft temperature difference due to the Morton effect. The method can be used for any type of journal bearing for which a typical steady state bearing design analysis code is available. The only special requirement of the bearing analysis code is that the steady state circumferential temperature profile of the lubricant be available for post-processing. Shaft temperature difference is calculated as follows:

1. For a selected combination of speed and load, run the bearing analysis program to get the static eccentricity position, and the stiffness and damping coefficients of the bearing.

2. Select a journal orbit for which to compute a shaft delta T. Either use an orbit of your choosing, or run a rotordynamic imbalance response analysis of the subject machine to calculate an orbit at the bearing. If the rotordynamic model contains a housing or bearing support, the orbit should be the relative orbit between the shaft and housing. As an example, figure 2 shows a journal orbit which is a circle.

3. Run the bearing analysis program again, but this time specify the position of the journal center to be the point corresponding to figure 1(a). This is the instant when the film thickness is at its smallest value. The bearing program produces a circumferential temperature profile around the bearing. At this instant in time, the shaft surface temperature profile is taken to be equal to the calculated oil film temperatures.

4. Repeat the calculation done in the preceding step with the journal positioned at the points depicted in figures 1(b-d). Note that as the journal travels around the orbit, it also rotates. For each of these positions the shaft surface circumferential temperature profile is calculated with the bearing program.

5. Upon completion of the preceding step, four values of temperature can be obtained for any point on the shaft surface at the four instances shown in Figure 1 (using interpolation when necessary). These four values are then averaged to yield an estimate of the steady state temperature of any point on the shaft surface.

In practice, the circumferential temperature profile should be calculated for a large number of points (e.g. 25 to 50) around the shaft surface, in order to generate a detailed profile of shaft surface temperature. Also, more than 4 orbit positions should be 
employed. Trials done with several bearing types suggest that 24 equally spaced points around the orbit may be optimum from the standpoint of resolution and efficiency of calculation.

In steps 3 and 4 above, the orbital path was taken to be the one calculated in the imbalance response analysis of step 2. An alternative approach is to use an orbit determined by multiplying the dynamic bearing load from step 2 times the bearing stiffness matrix. This load could then be input to the bearing analysis program so as to calculate the journal position and circumferential temperature profile. This would produce a somewhat different displacement orbit and temperature profile. This approach has also been thoroughly examined, and results will be presented in a forthcoming paper.

The steady state bearing analysis program employed for this work employs a fairly typical shaft surface boundary condition of uniform temperature. That temperature can be 1) input directly, 2) set equal to the circumferential average of the oil film temperature calculated during solution, or 3) set equal the value that results in zero net heat exchange between shaft and oil film. Calculation of the Morton Effect shaft temperature difference was not influenced significantly by the choice of this option.

Balbahadur and Kirk [12-14] have presented a method for estimating shaft temperature difference, and demonstrated its application to several case histories. Their method performs a time integration of a fluid film transient heat generation expression for 180 degrees of shaft revolution. A temperature rise value resulting from the integration is used to establish the shaft surface circumferential temperature profile on a point by point basis around the synchronous orbit of the journal. This method is similar, but still quite different, than the method presented here which only post-processes output from a standard bearing analysis program. Their method also does not compute an eigenvalue to assess stability, but instead focuses on the extent of the change in imbalance due to the estimate of temperature difference.

Kirk, Guo, and Balbahadur [15] have also presented results of shaft temperature difference measurements for what is referred to in their publication as an improved method of hot-spot location. Again, their method performs a time integration of an analytical fluid film transient heat generation expression (equation (9) in [15]) in order to derive the circumferential shaft temperature distribution. This transient method is quite different than the static method presented here where an industrial bearing design code is used to generate steady-state fluid film circumferential temperature profiles at discrete points in a synchronous orbit path. The temperature profiles are then transformed to the rotating shaft frame of reference and average temperatures computed at each location on the shaft in order to generate the final shaft temperature differential profile. 
The above calculation procedure utilizes a synchronous journal orbit taken from an imbalance response calculation done with a rotordynamic model. For evaluating both the $\vec{A}$ and $\vec{B}$ sensitivity vectors we used the average orbit radius since the orbit size must be expressed by a single quantity. We have identified 4 ways the orbit analysis could be done. Two use a displacement orbit with the bearing program being run with specified positions on the orbit to compute the temperatures. The other two use a force orbit with the bearing program being run with an applied static force.

1. Displacement orbit from imbalance response.

2. Assumed displacement orbit (e.g. a circle).

3. Force orbit from imbalance response.

4. Assumed force orbit (e.g. $10 \%$ of static load)

When using a displacement orbit (methods 1 or 2) the orbit is added to the static eccentricity vector, and the bearing program is executed in a "specified position" mode. When using a force orbit ( 3 or 4 ) the orbit is added to the nominal static journal load, and the bearing program is executed in a "specified load" mode. Methods 3 and 4 will also produce a displacement orbit that is statically derived as opposed to dynamic. All 4 methods will produce a value for shaft temperature difference. Regardless of which method is used, a single value for displacement orbit size must be selected for evaluating the $\vec{A}$ and $\vec{B}$ sensitivity vectors. We used method number 1 for the examples which follow because it clearly suggests using the same orbit size for both $\vec{A}$ and $\vec{B}$. Thus, when assessing stability with the product $\vec{B} \vec{A} \vec{C}$ the orbit size cancels.

\section{EXAMPLE CALCULATIONS OF SHAFT DELTA T}

As an example, the above method has been used to analyze the bearing studied by Gomiciaga and Keogh [5] who used CFD methods. The subject is a sleeve bearing with two axial feed grooves on the sides. This bearing was subject to extensive temperature and pressure measurements by Lund and Tonnesen [9], who also had thermocouples located in the shaft, but temperature difference across the shaft was not measured. The parameters of calculation case b) reported in [5] are as follows:

$\begin{array}{ll}\text { Shaft diameter: } & 100 \mathrm{~mm} \\ \text { Bearing outer diameter: } & 200 \mathrm{~mm} \\ \text { Bearing axial length: } & 55 \mathrm{~mm}\end{array}$

Pad arc: 171 degrees 


\begin{tabular}{|c|c|}
\hline Radial clearance: & $0.0726 \mathrm{~mm}$ \\
\hline Oil inlet temperature: & $50^{\circ} \mathrm{C}$ \\
\hline Oil density: & $850 \mathrm{~kg} / \mathrm{m}^{3}$ \\
\hline Oil specific heat: & $2000 \mathrm{~J} /(\mathrm{kg}-\mathrm{K})$ \\
\hline Oil viscosity at $\mathrm{T}$ inlet: & $0.0183 \mathrm{~Pa}-\mathrm{s}$ \\
\hline Oil viscosity exponent: & $0.02951 /{ }^{\circ} \mathrm{C}$ \\
\hline Oil supply flow rate: & 2.95 liter/min \\
\hline Bearing outer heat transfer coeffic & : $50 \mathrm{~W} /\left(\mathrm{m}^{2}-\mathrm{K}\right)$ \\
\hline Static eccentricity magnitude: & 0.7 \\
\hline Static eccentricity angle from verti & :38 degrees \\
\hline Circular orbit size, peak-to-peak: & $0.01452 \mathrm{~mm}$ \\
\hline Speed: & $10,000 \mathrm{rpm}$ \\
\hline In [5] the following calculated tem & atures were repo \\
\hline Peak babbitt temperature: & $125^{\circ} \mathrm{C}$ \\
\hline Peak shaft temperature: & $103.5^{\circ} \mathrm{C}$ \\
\hline Shaft temperature difference & $1.0^{\circ} \mathrm{C}$ \\
\hline
\end{tabular}

Calculation results were also given in [5] for backward circular whirl, and a slightly larger temperature difference of $1.2^{\circ} \mathrm{C}$ was reported. Keogh and Morton [2] analyzed a 360 sleeve bearing for circular forward whirl and backward whirl. They predicted a much higher difference for forward whirl than backward whirl $\left(10^{\circ} \mathrm{C}\right.$ and $\left.4^{\circ} \mathrm{C}\right)$. The calculation method here, however, cannot distinguish between forward and backward whirl. The orbit was processed with 24 equally spaced points, and temperature was determined at 24 equally spaced points on the shaft surface. Figure 3 shows the circumferential shaft surface temperature profile. The results are summarized as follows: 
Peak babbitt temperature:

Peak shaft temperature:

Shaft temperature difference $125^{\circ} \mathrm{C}$

$113^{\circ} \mathrm{C}$

$3.6^{\circ} \mathrm{C}$

The temporal lag of the hot spot behind the high spot of the whirling journal, reported as $38^{\circ}$ in [5], also cannot be determined here because the calculations are not truly transient. The calculated temperature difference was found to be quite linear with orbit size. Either increasing or decreasing the orbit size by a factor of two produced nearly an identical change in temperature difference.

As a second example, a double overhung turboexpander supported on 5 pad tilting pad bearings with maximum continuous operating speed (MCOS) of 18,600 rpm will be studied [10]. The initial design of this unit exhibited unstable Morton effect behavior starting at a speed near 18,000 rpm. This rotor, shown in Figure 4, has a very stiff shaft and runs well above 2 rigid body critical speeds (both below 10,000 rpm), and well below the first flexible rotor mode (near 30,000 rpm). The first flexible mode shape (figure 5) has maximum deflections at the two impellers. Since differential heating in either bearing will cause the nearby impeller to go out of balance, such a mode has potential to be unstable due to differential heating. This analysis focuses on the compressor end bearing, which is near the larger and heavier of the two impellers. Vibration at this bearing exhibited a divergent spiral during testing (see figure 6). The tilting pad bearing parameters are as follows:

$\begin{array}{ll}\text { Shaft diameter: } & 90 \mathrm{~mm} \\ \text { Bearing outer diameter: } & 117 \mathrm{~mm} \\ \text { Bearing axial length: } & 55 \mathrm{~mm} \\ \text { Pad arc: } & 52 \text { degrees } \\ \text { Pad pivot offset: } & 0.5 \\ \text { Pad preload: } & 0.35 \\ \text { Load direction: } & \text { on pad } \\ \text { Assembled diametral clearance: } & 0.185 \mathrm{~mm} \\ \text { Oil inlet temperature: } & 43^{\circ} \mathrm{C} \\ \text { Oil properties: } & \mathrm{ISO}^{2} 46 \\ \text { Oil supply flow rate: } & 23.6 \mathrm{liter} / \mathrm{min} \\ \text { Bearing outer heat Xfer coefficient: } & 50 \mathrm{~W} /\left(\mathrm{m}^{2}-\mathrm{K}\right)\end{array}$


The analysis was performed over a range of speeds. At each speed the stiffness and damping properties of the bearings were computed along with static eccentricity. Next, the imbalance response of the rotor to a concentrated imbalance at the compressor impeller was run to get the shaft orbit in the compressor end bearing at that speed. The shaft temperature difference is then calculated for that orbit. The imbalance response analysis yields $\vec{A}$, the balance sensitivity vector, which at MCOS is 0.05 micrometers peak to peak per gm-mm of imbalance. The phase angle of $\vec{A}$ is very close $180^{\circ}$ since the speed is far below the third mode (mode shape in figure 5). The bow coefficient vector, $\vec{C}$, was approximated using Eq (4), and is $67.9 \mathrm{gm}-\mathrm{mm}$ per degree Celsius of shaft temperature difference at the bearing. The phase angle of $\vec{C}$ is $180^{\circ}$ because the weight is outboard of the bearing. The temperature sensitivity vector $\vec{B}$ was calculated at each speed using the steady state bearing program and 24 points on both the shaft surface and orbit. For example, at $18,600 \mathrm{rpm}$ this was found to be $0.13{ }^{\circ} \mathrm{C} /$ micrometer. The size of the orbit used to determine this value is not important since the temperature was found to be linearly related to orbit size in a near perfect sense. The phase angle cannot be calculated with this analytical approach. A value of $20^{\circ}$ was used as this value was measured by De Jongh and Morton [11] for a 5 pad tilting pad bearing at 10,500 rpm. It is not known how much this phase angle varies versus speed since published measurements of this are not available. Based solely on the limited range of values of all available calculated and measured phase angles, it is believed to always be between $0^{\circ}$ and $60^{\circ}$ (lagging).

The product of the three vector magnitudes at $18,600 \mathrm{rpm}$ is seen to be only 0.44 (dimensionless). Therefore, being less than 1 , at this speed differential heating is predicted to be stable. Figure 7 shows the vector result for $\vec{B} \vec{A} \vec{C}$ as a function of speed. Instability is predicted to commence at approximately $21,500 \mathrm{rpm}$. As speed increases above this value, the instability becomes progressively stronger. Assuming a different value for the phase lag of vector $\vec{B}$ would rotate the curve in this plot about the origin, but the instability threshold would still be greater than $21,000 \mathrm{rpm}$, and this is considerably higher than that observed during unit testing. This discrepancy underscores that fact that the method of calculating temperature differential presented here, which appears promising, is but approximate and lacking of experimental verification. A future goal at the University of Texas is to conduct a test program to measure differential temperature in several bearing types in hopes of providing such verification. 


\section{CONCLUSIONS}

A simplified method of modeling bearing differential heating (aka, the Morton Effect) and its affect on shaft synchronous vibration has been presented. The presentation reveals that three rather elementary vectors, each of which serves to quantify a distinctly different type of machine sensitivity, combine in a simple way to dictate the stability, or lack thereof, of the Morton effect.

This method of analysis highlights the fact that the key missing ingredient to predicting the Morton effect instability for any rotating machine is $\vec{B}$, the temperature coefficient vector of sensitivity. It was suggested that straightforward testing of bearings for shaft differential heating (SDH) may provide the data needed to quantify $\vec{B}$ for a wide range of applications.

In addition, a new analytical method of estimating the magnitude of shaft differential temperature, $|\vec{B}|$, has been proposed. A key advantage of the method is it can be performed with a commonly available computer code for steady state fluid film bearing design. This potentially circumvents the need to perform a highly sophisticated three dimensional transient analysis of coupled rotordynamics, hydrodynamics and heat transfer. Some example calculations have been presented demonstrating the method.

\section{ACKNOWLEDGMENTS}

The authors thank Joseph Walch, of Cryostar SAS France, for providing material for the turboexpander example from [10].

\section{REFERENCES}

[1] Newkirk, B. L., 1926, “Shaft Rubbing,” Mechanical Engineering, 48, pp. 830-832.

[2] Keogh, P. S., and Morton, P. G., 1993, "Journal Bearing Differential Heating Evaluation with Influence on Rotor Dynamic Behaviour," Proceedings Roy. Soc. (London), Series A, Vol. 441, pp. 527-548.

[3] De Jongh, F. M., 2008, “The Synchronous Rotor Instability Phenomenon - Morton Effect," Proceedings of the 37th Turbomachinery Symposium, Turbomachinery Laboratory, Texas A\&M University, College Station, Texas.

[4] Andrisano, A. O., "An Experimental Investigation on the Rotating Journal Surface Temperature Distribution in a Full Circular Bearing," ASME Journal of Tribilogy, Vol. 110, October, 1988, pp. 638-645.

[5] Gomiciaga, R. and Keogh, P. S., 1999, “Orbit Induced Journal Temperature Variation in Hydrodynamic Bearings,” ASME Journal of Tribology, Vol. 121, January, 1999. 
[6] Dimarogonas, A. D. and Paipetis, S. A., 1983, Analytical Rotordynamics, Applied Science Publishers, New York.

[7] Larsson, B., "Heat Separation in Frictional Rotor-Seal Contact," ASME Journal of Tribology, Vol. 125, JULY 2003 , pp. 600-607.

[8] de Jongh, F.M. and van der Hoeven, P., 1988, "Application of a Heat Barrier Sleeve to Prevent Synchronous Rotor Instability," Proceedings of the 27th Turbomachinery Symposium, Turbomachinery Laboratory, Texas A\&M University, College Station, Texas.

[9] Lund, J. W., and Tonnesen, J., 1984, "An Approximate Analysis of the Temperature Conditions in a Journal Bearing. Part II: Application," ASME Journal of Tribology, Vol. 106, pp. 237-245.

[10] Schmied, J. S., Pozivil, J., and Walch, J., 2008, "Hot Spots in Turboexpander Bearings: Case History, Stability Analysis, Measurements and Operational Experience." Proceedings of ASME Turbo Expo 2008: Power for Land, Sea and Air, Berlin, Germany, June 9-13, 2008. ASME Paper Number GT2008-51179.

[11] De Jongh, F. M., and Morton, P. G., 1996, “The Synchronous Instability of a Compressor Rotor due to Bearing Journal Differential Heating,” ASME J. Eng. Gas Turbines Power, 118, pp. 816-824.

[12] Balbahadur, A., and Kirk, R. G., "Part I - Theoretical Model for A Synchronous Thermal Instability Operating in Overhung Rotors," IFToMM Sixth International Conference on Rotor Dynamics, UNSW, Sept., 2002.

[13] Balbahadur, A., and Kirk, R. G., "Part II - Case Studies for a Synchronous Thermal Instability Operating in Overhung Rotors," IFToMM Sixth International Conference on Rotor Dynamics, UNSW, Sept., 2002.

[14] Balbahadur, A., "A Thermoelastohydrodynamic Model of the Morton Effect Operating in Overhung Rotors Supported by Plain or Tilting Pad Journal Bearings," Virginia Polytechnic Institute and State University, Feb., 2001.

[15] Kirk, R. G., Guo, Z., and Balbahadur, A., "Synchronous Thermal Instability Prediction for Overhung Rotors," Proceedings of the $32^{\text {nd }}$ Turbomachinery Symposium, 2003. 
fig. 1. Four successive positions of an orbiting bearing journal within a sleeve bearing.

fig. 2. Examples of stable, borderline and unstable thermal spirals, respectively.

fig. 3. Shaft surface temperature profile computed for case b) from reference [4]. Mid-line of bearing journal.

fig. 4. Turboexpander presented in [9] used for second example calculation.

fig. 5. Rotordynamic model of turboexpander and mode shape of first flexible mode.

fig. 6. From fig. $6 \mathrm{~b}$ from [10] showing unstable divergent behavior in compressor end bearing at 18,600 rpm.

fig. 7. Morton stability chart of dimensionless vector $\vec{B} \vec{A} \vec{C}$ for turboexpander example, labels are rpm. 


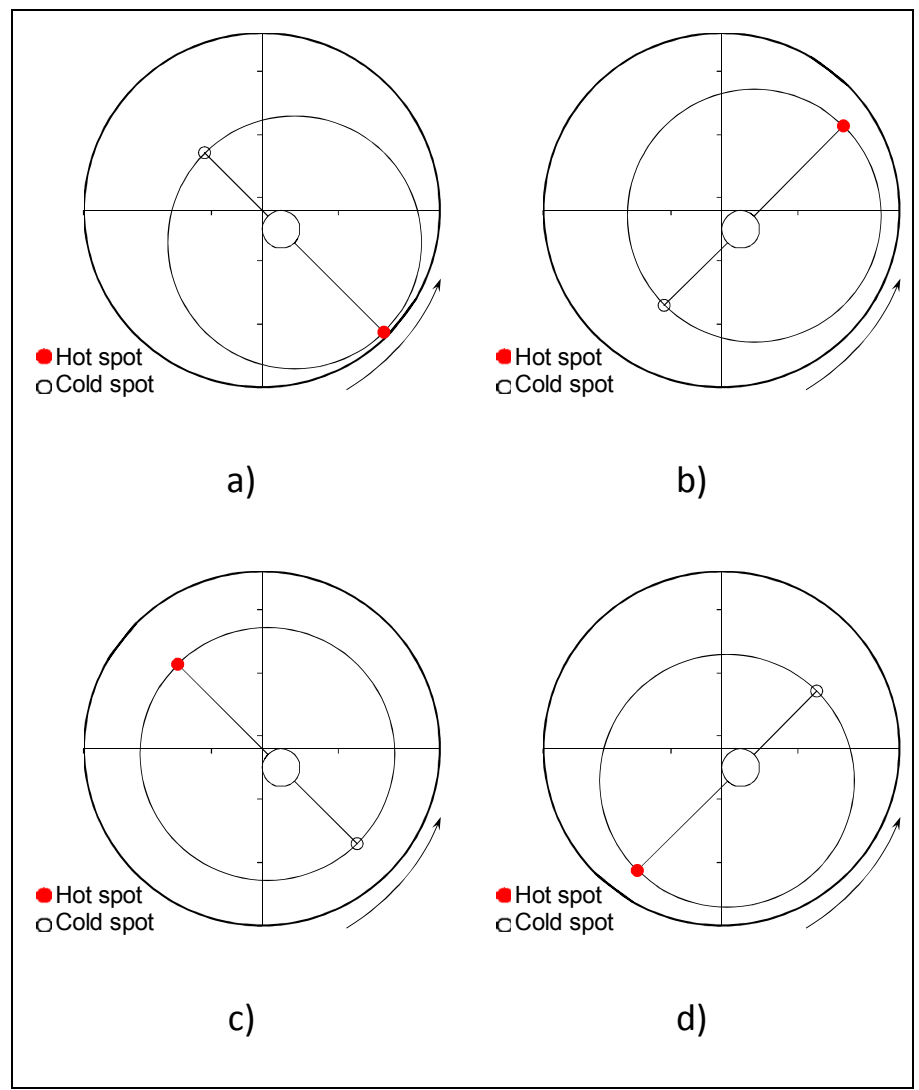

fig.1. Four successive positions of an orbiting bearing journal within a sleeve bearing. 

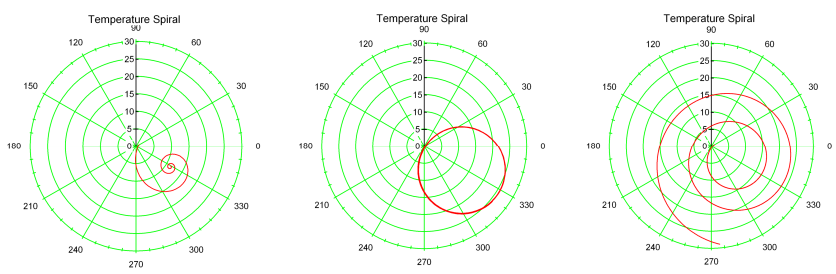

fig 2. Examples of stable, borderline and unstable thermal spirals, respectively. 


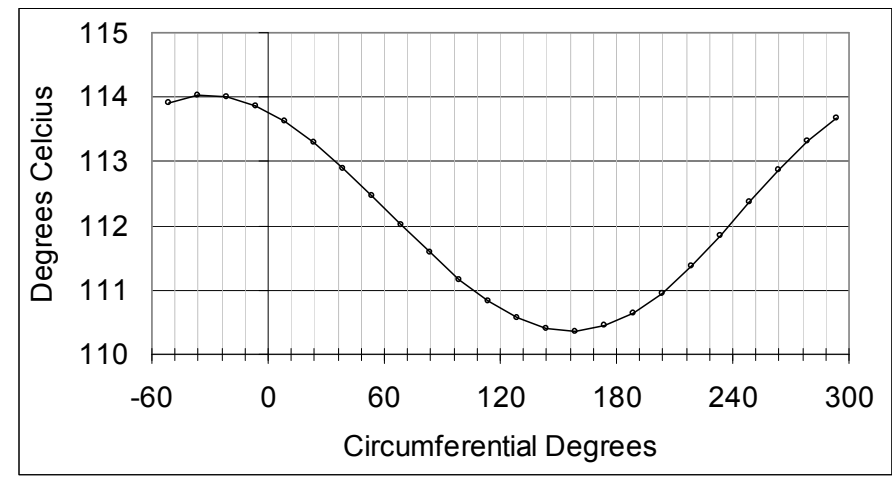

fig 3. Shaft surface temperature profile computed for case b) from reference [4]. Mid-line of bearing journal. 


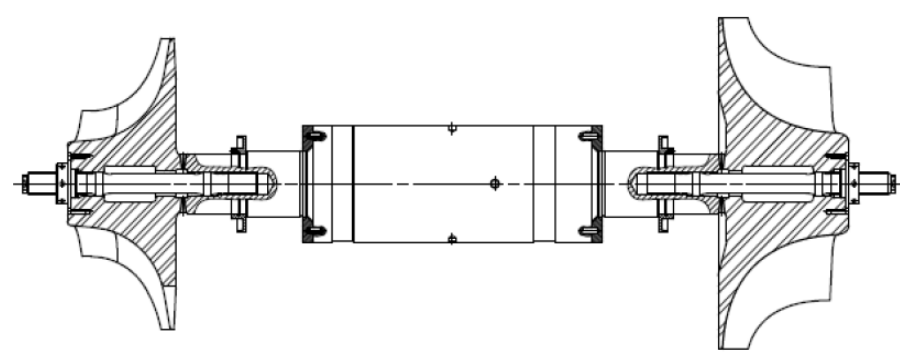

fig 4. Turboexpander presented in [9] used for second example calculation. 


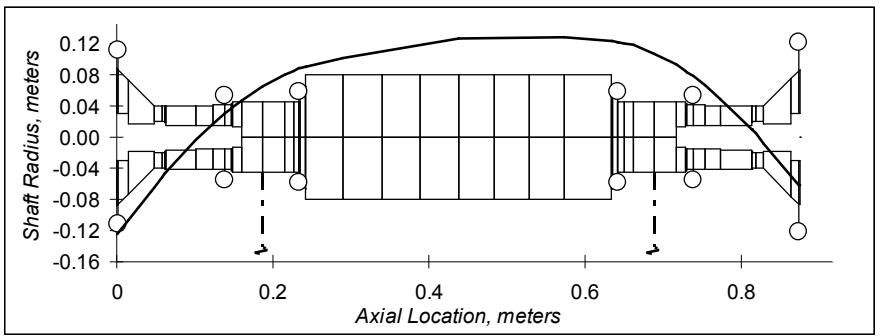

fig. 5. Rotordynamic model of turboexpander and mode shape of first flexible mode. 


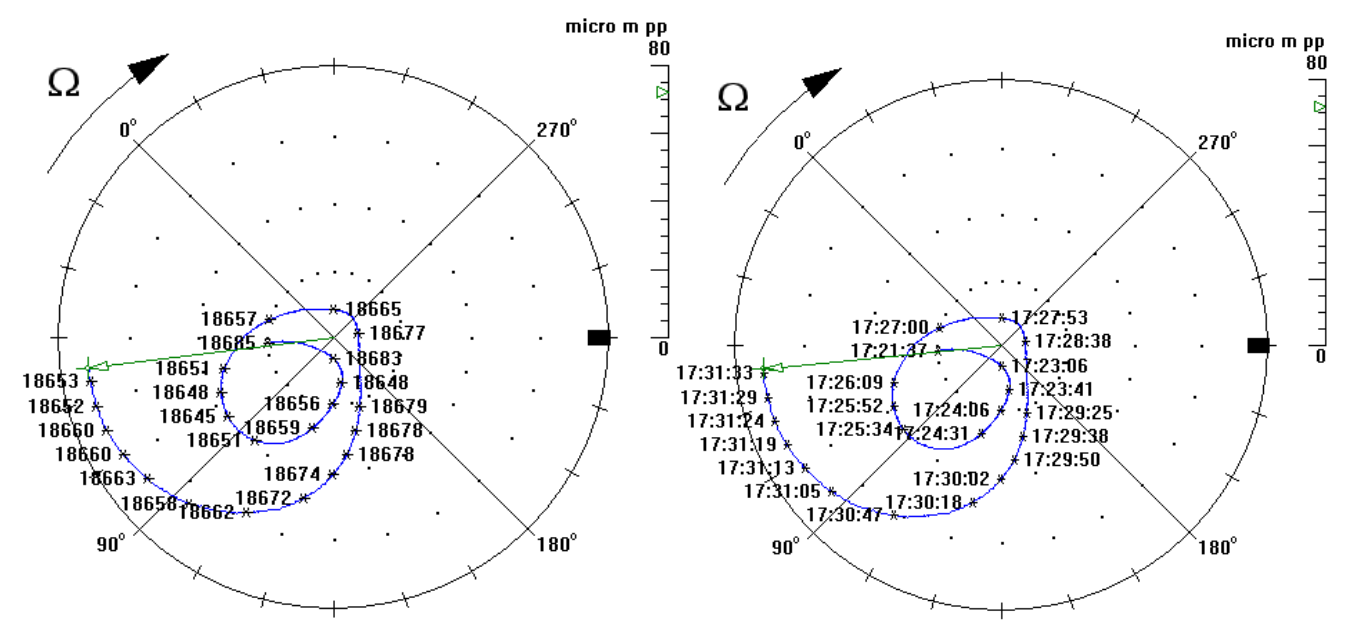

fig. 6. from fig. $6 \mathrm{~b}$ from [10] showing unstable divergent behavior in compressor end bearing at 18,600 rpm. 


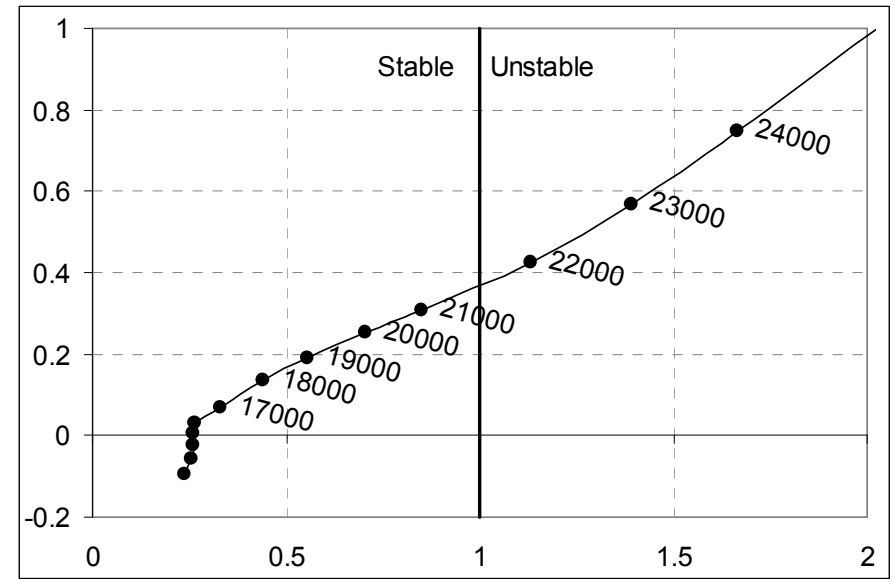

fig. 7. Morton stability chart of dimensionless vector $\vec{B} \vec{A} \vec{C}$ for turboexpander example, labels are rpm. 